\title{
How accurately can the SZ effect measure peculiar cluster velocities and bulk flows?
}

\author{
N. Aghanim ${ }^{1}$, K. M. Górski ${ }^{2,3}$, and J.-L. Puget ${ }^{1}$ \\ 1 IAS-CNRS, Bâtiment 121, Université Paris Sud, 91405 Orsay Cedex, France \\ 2 ESO, Karl-Schwarzschild-Str. 2, 85748 Garching bei München, Germany \\ 3 Warsaw University Observatory, Poland
}

Received 30 January 2001 / Accepted 4 May 2001

\begin{abstract}
The Sunyaev-Zel'dovich effect is a powerful tool for cosmology that can be used to measure the radial peculiar velocities of galaxy clusters, and thus to test, and constrain theories of structure formation and evolution. This requires, in principle, an accurate measurement of the effect, a good separation between the SunyaevZel'dovich components, and a good understanding of the sources contributing to the signal and their effect on the measured velocity. In this study, we evaluate the error in the individual radial peculiar velocities determined with Sunyaev-Zel'dovich measurements. We estimate, for three cosmological models, the errors induced by the major contributing signals (primary Cosmic Microwave Background anisotropies, Sunyaev-Zel'dovich effect due to the background cluster population, residuals from component separation and instrumental noise). We generalise our results to estimate the error in the bulk velocity on large scales. In this context, we investigate the limitation due to the Sunyaev-Zel'dovich source (or spatial) confusion in a Planck-like instrumental configuration. Finally, we propose a strategy based on the future all-sky Sunyaev-Zel'dovich survey, that will be provided by the Planck mission, to measure accurately the bulk velocities on large scales up to redshift 1 , or more.
\end{abstract}

Key words. cosmology: cosmic microwave background, large-scale structure of Universe - galaxies: clusters

\section{Introduction}

One of the major fields of research in cosmology is the study of the large scale matter distribution in the universe. On large scales, the evolution of the matter density fluctuations is adequately described through linear physics. The matter distribution thus represents the imprint of the initial density perturbations. Combined with other results, such as those derived from Cosmic Microwave Background $(\mathrm{CMB})$ observations, this kind of information can probe the structure formation models and the cosmological parameters.

The luminous matter distribution can be probed through the direct observation of galaxy, or galaxy cluster, distributions but this gives a biased view of the total matter distribution. The latter can be inferred from the velocity fields. In fact, the inhomogeneities in the mass distribution produce deviations from the Hubble flow referred to as the peculiar velocities. A few methods have been suggested to measure the transverse velocity components, e.g., Birkinshaw (1983) and Birkinshaw \& Gull (1983). However, in practice, the radial component of the peculiar velocities can only be measured using redshift

Send offprint requests to: N. Aghanim,

e-mail: nabila.aghanim@ias.u-psud.fr surveys, and by some established relationships which give independent distances to the objects, such as the FaberJackson (Faber \& Jackson 1976) or the Tully-Fisher (Tully \& Fisher 1977) relations. Nevertheless, these methods lead to uncertainties in the expansion velocity which are proportional to the distance and thus induce even larger uncertainties when measuring peculiar velocities on very large scales. The velocity fields can be studied using galaxies or galaxy clusters. However, there are more advantages in studying the deviations from the Hubble flow as traced by galaxy clusters. One of these advantages comes from the fact that, on scales probed by galaxy clusters, the underlying density fluctuations are largely in the linear regime and therefore very close to the initial conditions from which large scale structures developed. Finally, given observed radial peculiar velocities and the potential flow assumption, the full three-dimensional velocity field can be derived using reconstruction methods (Bertschinger \& Dekel 1989; Zaroubi et al. 1999 and references therein). The inferred density field is, in principle, representative of both the dynamical evolution of the structure and the underlying total matter distribution. Therefore, comparisons between the reconstructed total matter distribution from velocity fields and CMB fluctuations and that traced by 
galaxies can test and constrain theories of structure formation and evolution, and cosmological models.

Moreover, given cluster radial peculiar velocities at known positions, cluster motions can be used as cosmological probes using various statistical quantities among which are the velocity frequency distribution, the power spectrum, the velocity dispersions, the pairwise velocities, the velocity correlation function, and the bulk flows. As already mentioned, relative errors in the distance determination lead to the peculiar velocity uncertainty increasing linearly with distance. As a consequence, all the statistical quantities derived from observed velocities suffer from large errors especially at high redshifts. For example, in the case of the bulk velocity, which is the average of the local velocity field smoothed over a window function of scale $R h^{-1} \mathrm{Mpc}\left(h=H_{0} /\left(100 \mathrm{~km} \mathrm{~s}^{-1} / \mathrm{Mpc}\right)\right.$ is the normalised Hubble constant), several measurements exist in the literature (Dressler et al. 1987; Courteau et al. 1993; Willick et al. 1996; Giovanelli et al. 1998; Hudson et al. 1999; Willick 1999). Whereas most of these studies agree on the reality of a significant bulk flow within $50 h^{-1} \mathrm{Mpc}$; the situation is more controversial at larger scales where the accuracy of the determination of the bulk velocities decreases.

In order to overcome the problem of large uncertainties in the peculiar velocities, we must find ways of measuring them with a redshift independent accuracy. In this context, the Sunyaev-Zel'dovich (SZ) effect is a very promising, and potentially sensitive, tool. As proposed initially by Sunyaev \& Zel'dovich (1980), the radial peculiar velocity of galaxy clusters can be determined using SZ measurements, which are in addition distance independent. In the forthcoming years several experiments (ground based, balloon borne or space) will measure the SZ effect and perform SZ surveys (AMIBA, ARCHEOPS, BOLOCAM, MAP, ...). These experiments, of which the Planck satellite $^{1}$ is the best example, are designed so that the sensitivity, the frequency coverage and the angular resolution allow a very good separation of the two SZ effect components (thermal and kinetic, see Sect. 2), and a best possible evaluation of the foreground (galactic and extra-galactic) emissions that contribute to the measured signal. In this context, we expect that the number of observed SZ clusters will rapidly increase, thus allowing the radial peculiar velocity of clusters to be measured and providing a useful cosmological tool. This kind of project has already been undertaken on a sample of 40 known galaxy clusters with redshifts ranging between 0.1 and 0.3 by the SUZIE team (Holzapfel et al. 1997). Nevertheless, the accuracy in individual clusters is limited by the contamination from other components of the microwave sky. It has been suggested by Aghanim et al. (1997) and Kashlinsky \& Atrio-Barandela (2000) that averaging over many clusters in a large volume is probably the best method to measure the large scale velocity field up to $z=1$. This requires a sensitive all-sky SZ survey which will be provided only by the Planck mission.

\footnotetext{
${ }^{1}$ http://astro.estec.esa.nl/Planck
}

In this paper, we investigate quantitatively the accuracy of such a measurement, within the context of Planck.

As described in Sects. 2 and 3, the combination of the so-called kinetic and thermal SZ effects allows us to measure the radial peculiar velocity. However, this measurement is affected by errors due to astrophysical contributions (CMB, background cluster population), to residuals from the component separation and to instrumental noise. In Sect. 5, we present the method used to evaluate the rms error which affects the peculiar velocity measurement. We give, for three cosmological models, the results obtained for the major sources of error in terms of the rms error as a function of the cluster size for a Planck measurement. These errors are evaluated using simulated maps. The cluster model and the map simulation are presented in Sect. 4. In Sect. 6, we generalise the computation of the rms error to the bulk velocities at an illustrative scale of $100 h^{-1}$ Mpc. We discuss our results and conclude in Sect. 7 .

In the following, we use the baryon density $\Omega_{\mathrm{b}}=0.06$ (Walker et al. 1991). We discuss three cosmological models: an open model with a density parameter $\Omega_{\mathrm{m}}=0.3$, and two flat models: one with a non-zero cosmological constant $\left(\Omega_{\mathrm{m}}=0.3\right.$ and $\left.\Omega_{\Lambda}=0.7\right)$, and the other with no cosmological constant $\left(\Omega_{\mathrm{m}}=1\right)$.

\section{The SZ (thermal and kinetic) effect}

The SZ effect comprises the so-called thermal and kinetic effects. The thermal SZ effect is the inverse Compton interaction between CMB photons and the free electrons of the hot intra-cluster medium. Its amplitude is characterised by the Compton parameter $y$ - the integral of the pressure along the line of sight - which depends only on the cluster electron temperature and density $\left(T_{\mathrm{e}}, n_{\mathrm{e}}\right)$ :

$y=\frac{k \sigma_{\mathrm{T}}}{m_{\mathrm{e}} c^{2}} \int T_{\mathrm{e}}(l) n_{\mathrm{e}}(l) \mathrm{d} l$

where $k$ is the Boltzmann constant, $\sigma_{\mathrm{T}}$ the Thomson cross section, $m_{\mathrm{e}}$ the electron mass, $c$ the speed of light and $l$ is the distance along the line of sight. If the intra-cluster gas is isothermal $\left(T_{\mathrm{e}}(l)=T_{\mathrm{e}}=\right.$ const), $y$ is expressed as a function of the optical depth $\tau\left(\tau=\sigma_{\mathrm{T}} \int n_{\mathrm{e}}(l) \mathrm{d} l\right)$ :

$y=\tau \frac{k T_{\mathrm{e}}}{m_{\mathrm{e}} c^{2}}$

The inverse Compton interaction conserves the number of photons and shifts their spectrum, on average, to higher frequencies. This can be observed as the induced relative monochromatic intensity difference between Compton distorted and undistorted CMB:

$\frac{\Delta I_{\nu}}{I_{\nu}}=y \cdot f(x)$,

where $x$ is the dimensionless frequency $x=h_{\mathrm{pl}} \nu / k T_{\mathrm{CMB}}$ $\left(h_{\mathrm{pl}}\right.$ denotes the Planck constant, $T_{\mathrm{CMB}}$ the CMB temperature, and $\nu$ the frequency), $I_{\nu}$ is the intensity of the 
CMB (black body emission) and $f(x)$ is the spectral form factor given by:

$f(x)=\frac{x \mathrm{e}^{x}}{\left(\mathrm{e}^{x}-1\right)}\left[x\left(\frac{\mathrm{e}^{x}+1}{\mathrm{e}^{x}-1}\right)-4\right]$.

In the non-relativistic approximation, this spectral signature is universal, whereas it varies with the temperature of the intra-cluster gas in the exact computations including the relativistic corrections (Wright 1979; Rephaeli 1995; Challinor \& Lasenby 1998; Itoh et al. 1998; Pointecouteau et al. 1998; Sazonov \& Sunyaev 1998; Hansen \& Lilje 1999; Molnar \& Birkinshaw 1999; Nozawa et al. 2000).

When the cluster moves with a radial peculiar velocity $v_{\mathrm{r}}$, an additional relative intensity variation of the CMB due to the first-order Doppler effect is generated. It is:

$\frac{\Delta I_{\nu}}{I_{\nu}}=-\frac{v_{\mathrm{r}}}{c} \tau \times a(x)=\left(\frac{\Delta T}{T}\right)_{\mathrm{SZ}} \times a(x)$,

where $a(x)$ is a spectral form factor, given by:

$a(x)=x \frac{\mathrm{e}^{x}}{\mathrm{e}^{x}-1}$.

This is what is commonly referred to as the kinetic SZ effect. The intensity fluctuation induced by the kinetic SZ effect has the same spectral shape as the primordial anisotropies (equivalent to a temperature fluctuation). The amplitude of the temperature anisotropy induced by the kinetic SZ effect is thus:

$$
\left(\frac{\Delta T}{T}\right)_{\mathrm{SZ}}=-\frac{v_{r}}{c} \tau \text {. }
$$

The effect is positive for clusters moving towards the observer (i.e. with negative velocities).

\section{Measuring the peculiar velocity with SZ}

The characteristic spectral signature of the thermal SZ effect makes it a powerful tool for detecting galaxy clusters through millimetre and submillimetre observations. The kinetic SZ effect has a very different spectral signature from the thermal effect. In particular, it peaks at about $1.4 \mathrm{~mm}$ where the thermal effect is null. A multifrequency observation of a galaxy cluster should therefore allow a separation of the two effects. Consequently, the SZ effect can in principle be used as a tool for measuring the radial component of the galaxy cluster peculiar velocity $v_{\mathrm{r}}$. In fact, when we combine the thermal and kinetic SZ (Eqs. (2) and (3)), we obtain:

$v_{\mathrm{r}}=-c \frac{k T_{\mathrm{e}}}{m_{\mathrm{e}} c^{2}} \frac{(\delta T / T)_{\mathrm{SZ}}}{y}$.

This method was first suggested by Sunyaev \& Zel'dovich (1980), who also proposed to use it for bulk motion measurements at large scales. Rephaeli \& Lahav (1991) made one of the first estimates of the possibility of measuring the peculiar velocities using a selected sample of galaxy clusters. However, the most convincing measurements on individual clusters have only been done recently with the new generation of very sensitive bolometers (Holzapfel et al. 1997; Lamarre et al. 1998).

As mentioned in the previous section, the primary $\mathrm{CMB}$ anisotropies have the same spectral signature as the kinetic SZ effect of galaxy clusters. Therefore, the measured $(\delta T / T)$ towards a targeted cluster is contaminated by the primordial temperature fluctuations of the CMB. It is also contaminated by the background fluctuations induced by the clusters population, by any non-removed or residual astrophysical foreground contribution, and finally by the instrumental noise. These sources of contamination are responsible for an error $\delta v_{\mathrm{r}}$ in the estimated cluster peculiar velocity. When computed using Eq. (4), the relative error in the velocity can be expressed as follows:

$\frac{\delta v_{\mathrm{r}}}{v_{\mathrm{r}}}=\frac{\delta A}{A}+\frac{\delta T_{\mathrm{e}}}{T_{\mathrm{e}}}$

where $A=\frac{(\delta T / T)}{y}$. The $\delta T_{\mathrm{e}} / T_{\mathrm{e}}$ term is the relative error due to the uncertainty in the intra-cluster gas temperature which should be derived from X-ray data or from the SZ effect itself, as proposed by Pointecouteau et al. (1998). For the new generation of X-ray satellites (Chandra, XMMNewton), this error is expected to be of the order of 5 to $10 \%$. Hereafter, we will neglect this source of error in the evaluation of $\delta v_{\mathrm{r}}$. The error due to the CMB primary fluctuations enters into the term $A$ of Eq. (5) through the measurement of $\delta T / T$. In the same manner, the fluctuations generated by the background population of galaxy clusters through their kinetic SZ effect and all spurious emissions (astrophysical residuals, noise, ...) will also contribute to the error in $\delta v_{\mathrm{r}}$ through the $\delta T / T$ term. The residuals of the component separation between thermal and kinetic SZ effects should contribute either to the $\delta T / T$ term, or to the measurement of the cluster Compton parameter $y$. In our study, we take them into account as an additional $\delta T / T$ component.

Based on a map analysis, we evaluate the error in the peculiar velocity of individual galaxy clusters due to all contributing sources (CMB, clusters, ...) and we express it in terms of an induced rms error in the velocity. In this context, each source of error, $i$, will contribute in a quadratic form to the overall rms error, that is $\left(\delta v_{\mathrm{rms}}^{\text {tot }}\right)^{2}=$ $\sum_{i}\left(\delta v_{\mathrm{rms}}^{i}\right)^{2}$.

\section{Simulating the SZ effect of galaxy clusters}

We use a set of simulated maps for both the primary CMB fluctuations and the SZ effect contribution due to the thermal and the kinetic effects. All maps have $512 \times 512$ pixels of 1.5 arcmin square. We simulate the SZ effect using an empirical approach which consists of predicting the number of galaxy clusters that were formed between today and a redshift $z=10$. To do so, we use the Press-Schechter (PS) mass function (Press \& Schechter 1974). The individual galaxy clusters are modelled following a $\beta$-profile (see Sect. 4.2) and their positions on the simulated maps are drawn at random. 


\subsection{Cluster counts}

The general analytic expression for the PS counts gives the comoving number density of spherical collapsed halos in the mass range $[M, M+\mathrm{d} M]$ formed at a redshift $z$ :

$$
\begin{aligned}
\frac{\mathrm{d} n(M, z)}{\mathrm{d} M}= & -\sqrt{\frac{2}{\pi}} \frac{\bar{\rho}}{M^{2}} \frac{\mathrm{d} \ln \sigma(M, z)}{\mathrm{d} \ln M} \frac{\delta_{\mathrm{c} 0}(z)}{\sigma(M, z)} \\
& \times \exp \left[-\frac{\delta_{\mathrm{c} 0}^{2}(z)}{2 \sigma^{2}(M, z)}\right]
\end{aligned}
$$

where $\bar{\rho}$ is the mean comoving background density and $\delta_{\mathrm{c} 0}(z)$ is the overdensity of a linearly evolving structure. The mass variance $\sigma^{2}(M, z)$ of the fluctuation spectrum, filtered on mass scale $M$, is related to the power spectrum of the initial density fluctuations $P(k)$ (Peebles 1980). Following Viana \& Liddle (1996) and Viana \& Liddle (1999), we use an approximation of the variance in spheres of radius $R\left(R=(3 M / 4 \pi \bar{\rho})^{1 / 3}\right)$ in the vicinity of $8 h^{-1} \mathrm{Mpc}$ :

$$
\sigma(R, z)=\sigma_{8}(z)\left(\frac{R}{8 h^{-1} \mathrm{Mpc}}\right)^{-\gamma(R)}
$$

with

$$
\gamma(R)=(0.3 \Gamma+0.2)\left[2.92+\log \left(\frac{R}{8 h^{-1} \mathrm{Mpc}}\right)\right] .
$$

$\Gamma$ is the so-called shape parameter of the cold dark matter transfer function, taken to be 0.23 (see Viana \& Liddle 1999 for a discussion). The redshift evolution $\sigma_{8}(z)$ is given by the perturbation growth law (Carroll et al. 1992).

\subsection{Modeling individual clusters}

The spatial distribution of the SZ (thermal and kinetic) effect is ruled by the intra-cluster gas profile. The latter is generally well-described by the so-called $\beta$-profile (King 1966). We will therefore use for simplicity, as in Cavaliere \& Fusco-Femiano (1978), the hydrostatic isothermal model with a spherical geometry. In this model the electron density distribution is given by:

$n_{\mathrm{e}}(R)=n_{\mathrm{e} 0}\left[1+\left(\frac{R}{R_{\mathrm{c}}}\right)^{2}\right]^{-\frac{3 \beta}{2}}$,

where $n_{\mathrm{e} 0}$ is the central electron density, $R_{\mathrm{c}}$ is the cluster core radius and $\beta$ is a parameter whose value is about $2 / 3$ as indicated by both numerical simulations (Evrard 1990) and X-ray surface brightness profiles (Jones \& Forman 1984; Edge \& Stewart 1991). The physical parameters of a galaxy cluster (temperature, virial radius and central electron density) can be computed once its formation redshift and its mass are known. The cluster temperature $T_{\mathrm{e}}$, in $\mathrm{keV}$, is given by Bryan \& Norman (1998):

$T_{\mathrm{e}}=1.39 \frac{f_{\mathrm{T}}}{b} M_{15}^{2 / 3}\left[h^{2} \Delta_{\mathrm{c}}(z) E(z)^{2}\right]^{1 / 3}$,

where $f_{\mathrm{T}}$ and $b$ are numerical factors set respectively to 0.79 and $1, M_{15}$ is the cluster mass in $10^{15} M_{\odot}$ units, $\Delta_{\mathrm{c}}(z)$ is the critical density (expressions can be found in Bryan $\&$ Norman 1998) and $E(z)$ is related to the time by $t(z)=$ $H_{0}^{-1} \int_{z}^{\infty}(1+z)^{-1} E(z)^{-1}$ (see Peebles 1993 for example).

The central density $n_{\mathrm{e} 0}$ can be derived from the cluster gas mass using the following equation:

$M_{\text {gas }}\left(\frac{\Omega_{\mathrm{b}}}{\Omega_{\mathrm{m}}}\right)=m_{\mathrm{p}} \mu \int_{0}^{R_{\mathrm{vir}}} n_{\mathrm{e}}(R) 4 \pi R^{2} \mathrm{~d} R$,

where the virial radius of the structure, for a critical universe $\left(\Omega_{\mathrm{m}}=1\right)$, is given by:

$R_{\mathrm{vir}}=\frac{(G M)^{1 / 3}}{\left(3 \pi H_{0}\right)^{2 / 3}} \frac{1}{1+z}$.

In this equation, $m_{\mathrm{p}}$ is the mass of the proton, $\mu=0.6$ is the mean molecular weight of a plasma with primordial abundances, and $G$ is the gravitational constant. We can define the core radius of a cluster as $R_{\mathrm{c}}=R_{\mathrm{vir}} / p$, and arbitrarily set $p=15$ for all the clusters. Using these quantities and assuming the $\beta$-profile, we compute the profile of the $y$ parameter (Eq. (1)) associated with each cluster. The cluster atmosphere could depart from the spherical assumption made in our study. The most extreme geometrical variation would occur if the cluster is oblate or prolate with its unique axis oriented along the line of sight. Assuming typical ellipticities (see discussion in Birkinshaw et al. 1991 and Hughes \& Birkinshaw 1998 and references therein), the Compton parameter could be, at the very most, multiplied by a factor 0.5 to 2 due to the cluster asphericity. The second physical assumption we make concerns the intra-cluster gas distribution. There is still no strong evidence of a temperature decrease with radius (at large radii) from recent X-ray observations (apart from cooling flow and merger clusters). Chandra results, with large error bars, are consistent with a slightly decreasing temperature profile (Markevitch et al. 2000) whereas XMM-Newton higher precision observations show a "flat" profile consistent with the isothermal assumption (Arnaud et al. 2001) at least up to $0.7 R_{\text {vir }}$. Therefore in our study, we make the conservative assumption of isothermality.

The kinetic SZ anisotropy map is obtained from the thermal SZ map by introducing the radial component of the cluster peculiar velocity. In the assumption of an isotropic Gaussian distribution of the initial density perturbations, the initial power spectrum $P(k)$ gives a complete description of the velocity field through the three-dimensional rms velocity $\sigma_{\mathrm{v}}$ predicted by the linear gravitational instability at a scale $R$. This velocity is given by:

$\sigma_{\mathrm{v}}=a(t) H(t) f\left(\Omega_{\mathrm{m}}, \Lambda\right)\left[\frac{1}{2 \pi^{2}} \int_{0}^{\infty} P(k) W^{2}(k R) \mathrm{d} k\right]^{1 / 2}$

where $a(t)$ and $H(t)$ are respectively the expansion parameter and the Hubble constant. $W$ is the Fourier transform of the window function over which the variance is smoothed. The function $f\left(\Omega_{\mathrm{m}}, \Lambda\right)$ can always be approximated by $f\left(\Omega_{\mathrm{m}}, \Lambda\right)=\Omega_{\mathrm{m}}^{0.6}$ (Peebles 1980; 
Lahav et al. 1991). Furthermore in the assumption of linear regime and a Gaussian distribution of the density fluctuations, the structures move with respect to the Hubble flow with peculiar velocities, $v$, following a Gaussian distribution $f(v)=\frac{1}{\sigma_{\mathrm{v}} \sqrt{2 \pi}} \exp \left(\frac{-v^{2}}{2 \sigma_{\mathrm{v}}^{2}}\right)$ which is fully described by $\sigma_{\mathrm{v}}$. This prediction is in agreement with numerical simulations (Bahcall et al. 1994; Moscardini et al. 1996). We therefore compute the three-dimensional rms peculiar velocity using Eq. (11) for our three cosmological models. At $z \simeq 0$, we find it ranges between 400 and $500 \mathrm{~km} \mathrm{~s}^{-1}$ (Fig. 6), in good agreement with the observed velocity interval $300<v_{\mathrm{rms}}<700 \mathrm{~km} \mathrm{~s}^{-1}$ (Hudson 1994; Giovanelli et al. 1996; Moscardini et al. 1996). Our values, especially at low redshifts, could be underestimated by a factor of up to $40 \%$ due to the cluster non-linear evolution at latetime growth (Colberg et al. 2000). Nevertheless, we do not correct for it. We will see in the next section (Fig. 1) that the expected effect will remain negligible compared to the other contributions to the error in the peculiar velocity. Assuming a random distribution of angles, the amplitude of the peculiar velocity for individual clusters is drawn at random from the specified Gaussian distribution, and it is then assigned to the clusters one by one.

\section{The error in individual cluster peculiar velocities}

\subsection{Method}

The radial component of the galaxy cluster peculiar velocity can be determined using the ratio of thermal to kinetic SZ effect combined with the intra-cluster temperature. This requires a good separation of the thermal and kinetic components of the SZ effect which can be achieved, in principle, through accurate measurements at two or more millimetre/submillimetre wavelengths (Hobson et al. 1998; Bouchet \& Gispert 1999) as expected for the Planck mission.

Astrophysical or instrumental contributions will introduce systematic errors into the determination of the peculiar velocity $v_{\mathrm{r}}$. In this context, we examine the contributions of the background population of galaxy clusters, and then that of both the SZ clusters, and the CMB primary anisotropies. The results are given for three cosmological models using simulated maps convolved with a Gaussian beam of 5 arcmin (the Planck effective resolution for SZ measurements).

The CMB primary temperature fluctuations dominate down to angular scales of about 5 arcmin with amplitudes varying with scale and cosmological model. More generally, each contributing source to the signal (astrophysical or instrumental) has its own particular distribution in the amplitude-scale space. Therefore, the measurement of the SZ effect at a cluster scale (a few arcminutes to a few degrees) picks up a spurious signal from all the contributions at scales up to the cluster scale (typically the angular scale corresponding to its virial radius).
Following Aghanim et al. (1997), our method consists of detecting the cluster through its thermal SZ effect and measuring the kinetic SZ effect $(\delta T / T)$ at this position. The accuracy of the peculiar velocity determination depends on the accuracy of both of these measurements. We compute the error to the peculiar velocity due to the $\delta T / T$ term using a spatial filter. The filter is characterised by two windows: a central disk (centred on the cluster position) and an external ring, and thus by three parameters (the radius of the central disc, and the inner and outer radii of the ring). The optimum filter is chosen, through the thermal SZ effect measurement, using only the data, i.e. for the Planck resolution, the observed cluster profile (convolved with the Planck beam). In practice, due to beam dilution effects, we have obtained an optimum spatial filter that can be applied to a wide range of clusters. In this case, the disc corresponds to the region where the Compton parameter is greater than $70 \%$ of the observed central value, and the ring is defined by its inner radius $0.5 \times F W H M$ and its width $\Delta R=2$ pixels.

The peculiar velocity of a targeted cluster is then obtained by computing the ratio of the difference of the thermal and kinetic components of the SZ effect, between the two windows of the filter. Consequently, on a "blank field" containing foreground or background contributions (CMB, or cluster population, or galactic residuals), the filter is used to measure the expected contamination due to some, or all of these contributions, to the $\delta T / T$ term which is expressed in terms of an error $\delta v_{\mathrm{r}}$ in the peculiar velocity. In our case and due to the beam dilution, the contribution to the error in the peculiar velocity associated with the amplitude of the thermal SZ effect just acts as a normalisation factor $\delta v_{\text {rms }} \propto 1 / y$. In all the following results shown in Figs. 1-4, the errors are computed for a cluster whose central Compton parameter is $y_{0} \simeq 10^{-4}$.

To evaluate the accuracy of the peculiar velocity determination, we use the optimum spatial filter. We compute the differential signal in the filter windows and infer the induced error for individual clusters $\delta v_{\mathrm{r}}$ using many random positions on the simulated maps, convolved by a 5 arcmin beam. We obtain errors $\delta v_{\mathrm{r}}$ in 300 different positions from which we derive the rms error in the velocity, $\delta v_{\text {rms }}$. This operation is repeated for different cluster sizes (expressed in terms of the core radius) as we expect that the spurious contributions vary with the angular scale.

\subsection{Results}

We first analyse simulated maps of the background fluctuations induced by the kinetic SZ effect of a synthetic population of galaxy clusters. In order to separately estimate the contribution to the error from the background SZ kinetic fluctuations, no other contributing source is taken into account at this stage of the analysis. We plot, in Fig. 1, the obtained $\delta v_{\text {rms }}$ as a function of the cluster core radius for three cosmological models. As a general trend for all the cosmological models, the rms error in 


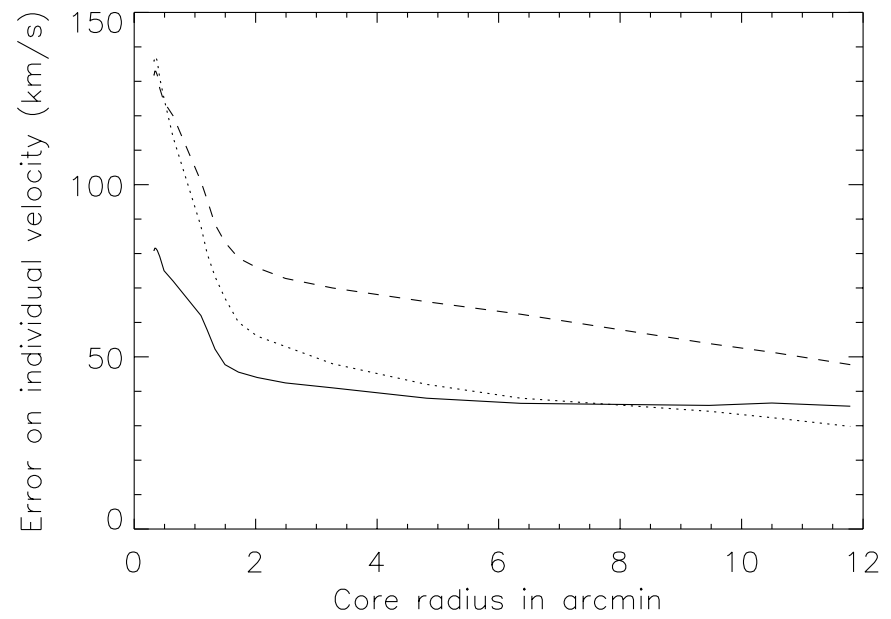

Fig. 1. The rms error in the peculiar velocity due to the kinetic SZ secondary anisotropies of galaxy cluster background population. The error is given for an individual galaxy cluster with $y_{0} \simeq 10^{-4}$ as a function of its core radius in arcmin and for three cosmological models. The solid, dotted and dashed lines represent respectively the standard model $\left(\Omega_{\mathrm{m}}=1, \Omega_{\Lambda}=0\right)$, the open model $\left(\Omega_{\mathrm{m}}=0.3\right)$ and the flat model with a cosmological constant $\left(\Omega_{\mathrm{m}}=0.3, \Omega_{\Lambda}=0.7\right)$.

the individual peculiar velocity decreases rather abruptly with increasing core radius for clusters with core radii up to 2 arcmin; whereas for larger radii the uncertainty decreases very slowly. This variation of the rms error with cluster size can be understood by the fact that, for extended clusters (i.e., large core radii), the temperature fluctuations (around zero) induced by the kinetic SZ effect of the background clusters are averaged out. Whereas for small core radii, the effect of beam dilution dominates. When we compare the error as a function of the cosmological model, we note that $\delta v_{\text {rms }}$ is the lowest for the standard model with no cosmological constant $\left(\Omega_{\mathrm{m}}=1\right.$, solid line). It ranges between less than $90 \mathrm{~km} \mathrm{~s}^{-1}$ (at maximum) and about $40 \mathrm{~km} \mathrm{~s}^{-1}$ for the largest core radii. In the open model $\left(\Omega_{\mathrm{m}}=0.3\right.$, dotted line $)$, the error reaches about $150 \mathrm{~km} \mathrm{~s}^{-1}$ for very small core radii, and can be as low as about $30 \mathrm{~km} \mathrm{~s}^{-1}$ for extended clusters. This is due to the combined effect of the cluster higher number counts, especially at high redshifts, and the fact that the velocity dispersions are higher in an open model. In the flat model with a non-zero cosmological constant $\left(\Omega_{\mathrm{m}}=0.3\right.$, $\Omega_{\Lambda}=0.7$, dashed line), the cluster peculiar velocities and the number of sources are larger than in the other models. As a consequence of the late-time non-linear growth of the galaxy clusters, the predicted error due to the kinetic SZ background population could be, roughly, up to $40 \%$ larger than the quoted numbers.

In the second step, we analyse a simulated $\delta T / T$ map containing both the primary CMB anisotropies and the kinetic SZ fluctuations due to the population of galaxy clusters studied above. The first thing we note in comparing Figs. 1 and 2, is that the primary CMB dominates the uncertainties, it induces larger errors than the

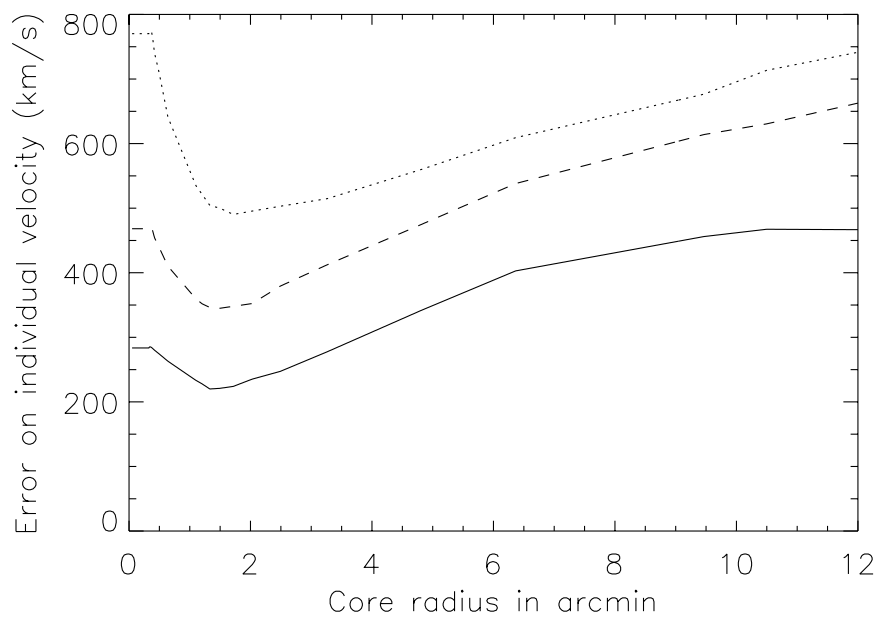

Fig. 2. For a $y_{0} \simeq 10^{-4}$ individual cluster with a core radius varying between 0 and 12 arcmin, we plot the rms error in the radial component of the peculiar velocity due to the kinetic SZ secondary anisotropies of the galaxy cluster background population plus the CMB primary anisotropies. The line-styles represent the same cosmological models as in Fig. 1.

SZ kinetic population and it has a different behaviour. In our three cosmological models, unlike the SZ background contribution, the CMB contribution increases in amplitude with increasing cluster size. In fact, $\delta v_{\text {rms }}$ decreases (beam dilution effect) and then increases with increasing core radius with a minimum at about 1.5 arcmin. This arises from the fact that the amplitude of the primary temperature fluctuations increases with the angular scale in the range sampled by our spatial filter (acoustic peak). Consequently, this larger contribution to $\delta T / T$ induces larger errors in the velocity at large core radii. The open model (dotted line) exhibits the largest errors which reach about $800 \mathrm{~km} \mathrm{~s}^{-1}$, with a minimum of about $500 \mathrm{~km} \mathrm{~s}^{-1}$ near 1.5 arcmin. In the flat model with a nonzero cosmological constant (dashed line), the errors culminate at about $650 \mathrm{~km} \mathrm{~s}^{-1}$ for extended clusters their minimum is about $350 \mathrm{kms}^{-1}$ near 2 arcmin and they reach about $450 \mathrm{~km} \mathrm{~s}^{-1}$ for small core radii. The standard model $\left(\Omega_{\mathrm{m}}=1\right.$, solid line), predicts the smallest error with a maximum, for extended clusters, of about $450 \mathrm{~km} \mathrm{~s}^{-1}$ a minimum at $200 \mathrm{~km} \mathrm{~s}^{-1}$ and an error of about $300 \mathrm{~km} \mathrm{~s}^{-1}$ for the smallest core radii. In this context, the contribution from the non-linear evolution of clusters is negligible with respect to the CMB primary contribution. The linear approximation used will thus almost not affect our results and conclusions.

So far, we have studied two cosmological signals (CMB and kinetic SZ) that contribute as sources of error in the velocity determination, and that are not separated (they have the same spectral signatures). In practice, the thermal SZ maps contain information on the position and shape of galaxy clusters that helps us in measuring the peculiar velocities. We have used this information in a very simple way by associating the position of the 


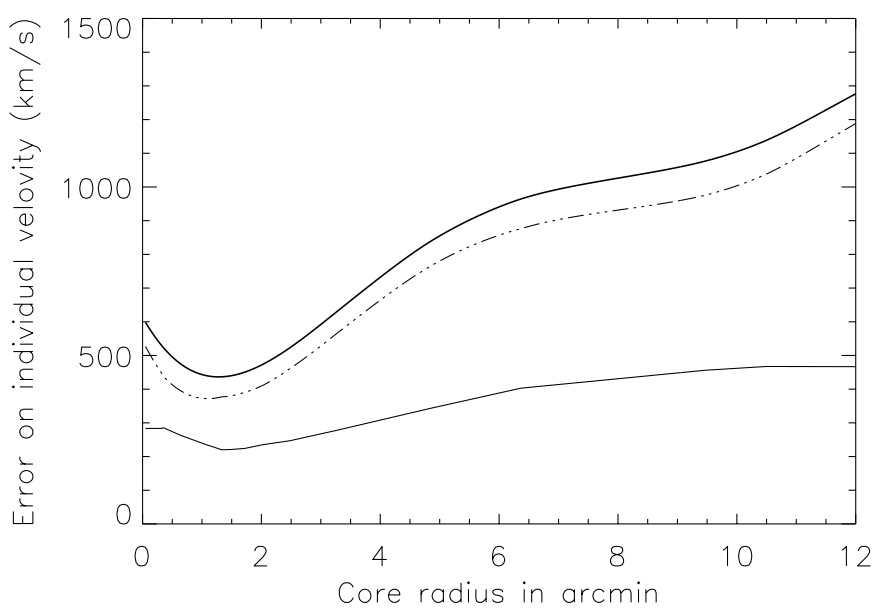

Fig. 3. The dotted long-dashed line represents the contribution to the rms error in the peculiar velocity due to the astrophysical residuals from the component separation and to the Plancklike instrumental noise (other than CMB and SZ). The thin solid line stands for the contribution of CMB and SZ kinetic background population in an $\Omega_{\mathrm{m}}=1$ model. The thick solid line represents the total rms errors.

maximum detected thermal SZ signal with the central position of the cluster, and also by optimising the spatial filter using the observed thermal map. More sofisticated methods have been proposed to use the additional phase information of the different signals in order to reduce the error due to the CMB fluctuations (Scherrer et al. 1991; Naselsky et al. 2000). This kind of method, applied for the best case of point-like sources (Naselsky et al. 2000), has shown its usefulness in detecting and extracting the point sources buried in a CMB signal. The efficiency of such a method, in reducing the CMB contribution to the peculiar velocity, for resolved sources convolved with the instrumental beam (as in our case) remains to be studied. In addition, our analysis has been done in an ideal way because we did not take into account the fact that a $\delta T / T$ map will result from a component separation. In this context, we expect that the residuals from the component separation will contribute to the error in the peculiar velocity. Among the residuals, an important contribution comes from the instrumental noise, which in the case of Planck, is about $2 \times 10^{-6} \mathrm{rms} \delta T / T$. For a standard model with $\Omega_{\mathrm{m}}=1$, we derive, from a previous study (Aghanim et al. 1997), the rms error due to the instrumental noise and the residual signals from the component separation (Fig. 3, dotted long-dashed line). The component separation was performed using Wiener filtering (Bouchet \& Gispert 1999), and the residuals account for the galactic contributions and the instrumental noise. The total $\delta v_{\text {rms }}$ (including the contribution from the CMB and kinetic SZ effect (Fig. 2, solid line)) is represented by the thick solid line in Fig. 3. This error in the peculiar velocity due to the residuals remaining after the component separation and to instrumental noise, is thus mostly independent of the cosmological model, and it should be present in the

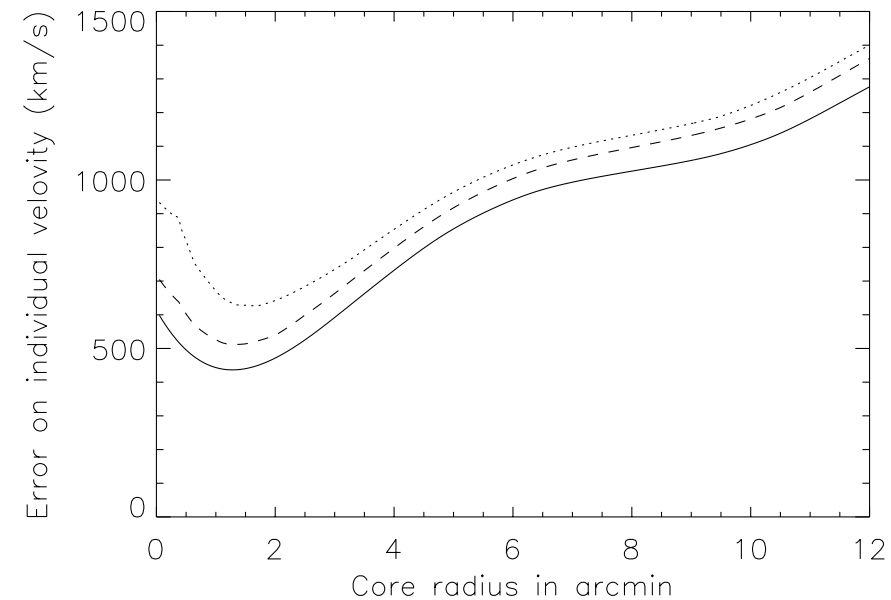

Fig. 4. The total rms error in the individual velocity due to all sources of confusion (CMB, background kinetic SZ, galactic residuals, residuals of component separation, Planck-like instrumental noise). The line-styles stand for the same cosmological results as in Fig. 1.

Planck measurements of the peculiar velocities using the SZ effect at this level, independent of the cosmological model. Furthermore, the induced error is dominant for the standard model, and dominant or comparable to the $\mathrm{CMB}+\mathrm{SZ}$ error in the other cosmological models. In this context, the phase information will not help and we do not expect a reduction of the total error in the velocity. The resulting total rms errors on the velocity of individual clusters are displayed in Fig. 4 where the solid line represents the standard $\left(\Omega_{\mathrm{m}}=1\right.$ model $)$, and the dotted and dashed lines stand respectively for the open and flat models.

\section{Bulk velocities}

The error in the individual velocities of galaxy clusters are rather large due to several contributions. However, we can obtain meaningful and valuable information on the velocity fields through statistical analyses. Over large scales, one accessible piece of statistical information derived from the cluster peculiar velocity is the bulk velocity. It is defined as the centre-of-mass velocity of a specified region, and it is given by the integral of the peculiar velocities over a selected volume specified by a selection function. In a given volume containing $N$ clusters with individual peculiar velocities $v_{i}$ each measured with an accuracy $\sigma_{i}$, the best estimate of the bulk velocity $V_{\text {bulk }}$ is the mean weighted velocity:

$V_{\mathrm{bulk}}=\frac{\sum_{i=0}^{N} \frac{1}{\sigma_{i}^{2}} v_{i}}{\sum_{i=0}^{N} \frac{1}{\sigma_{i}^{2}}}$ 


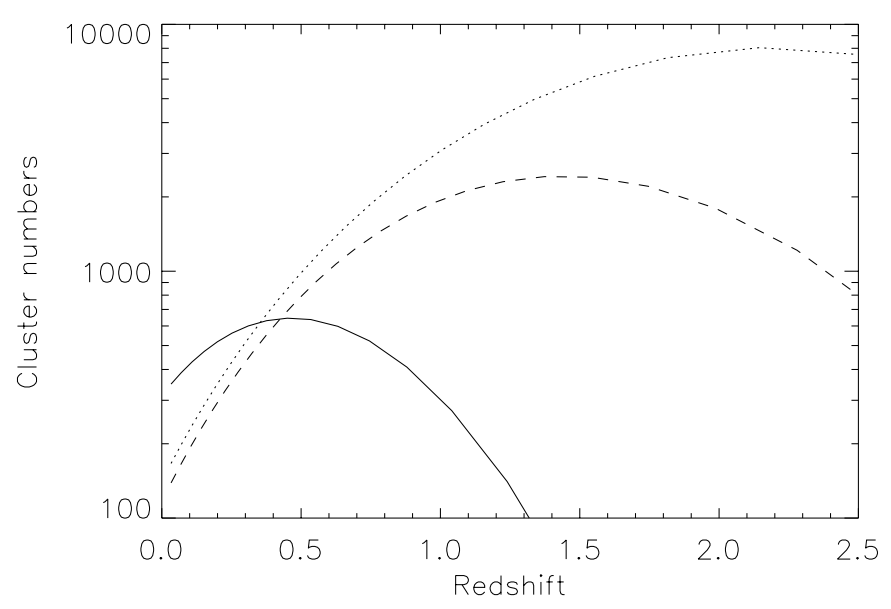

Fig. 5. The number of clusters as a function of redshift in the $100 h^{-1}$ Mpc size boxes. The solid line represents the standard model $\left(\Omega_{\mathrm{m}}=1\right)$, the dotted line represents the open (low $\Omega_{\mathrm{m}}$ ) model and finally the dashed line shows the results for a flat model with a non-zero cosmological constant.

We compute the error due to the astrophysical and instrumental contributions, in the bulk velocity, using the following relation:

$$
\sigma_{\text {bulk }}^{2}=\frac{1}{\sum_{i} \frac{N_{i}}{\sigma_{i}^{2}}},
$$

where $N_{i}$ is the number of clusters of mass $M_{i}$ and accuracy $\sigma_{i}$ at a given redshift.

In order to avoid local non-linear or correlation effects, we focus on scales that are large enough to allow a significant measurement of the bulk velocity. We choose an illustrative $100 h^{-1} \mathrm{Mpc}$ scale and investigate, for redshifts between 0 and 2, the accuracy of the bulk velocities when we take into account the major sources contributing to the error (CMB primary fluctuations, kinetic SZ fluctuations of a background cluster population, residuals due to component separation process and instrumental noise). In each "box" of $100 h^{-1}$ Mpc size defined by its $\Delta \Omega$ and $\Delta \mathrm{z}$ at redshift $z$, we use the PS number counts to compute the predicted number of clusters. For each of these clusters, we compute the core radius $\theta_{\mathrm{c}}$ and the central Compton parameter $y_{0}$. Thus using the results displayed in Fig. 4, we can associate with each cluster in a given volume an error in its individual peculiar velocity. Finally using Eq. (13), we compute the overall accuracy $\sigma_{\text {bulk }}$ in each $100 h^{-1}$ Mpc typical size volume and show the results as a function of redshift in Fig. 7 .

In the standard model, the bulk velocity at the $100 h^{-1}$ Mpc scale can be affected by an error as large as $400 \mathrm{~km} \mathrm{~s}^{-1}$ at small redshifts. This error decreases with redshift down to about $150 \mathrm{~km} \mathrm{~s}^{-1}$ by $z=0.8$. It remains almost constant until $z \simeq 1.3$ and increases again to very large values by $z=2$. This increase is associated with the lack of clusters at high redshifts in high $\Omega_{\mathrm{m}}$ models (see Fig. 5). In an open model the number of clusters being

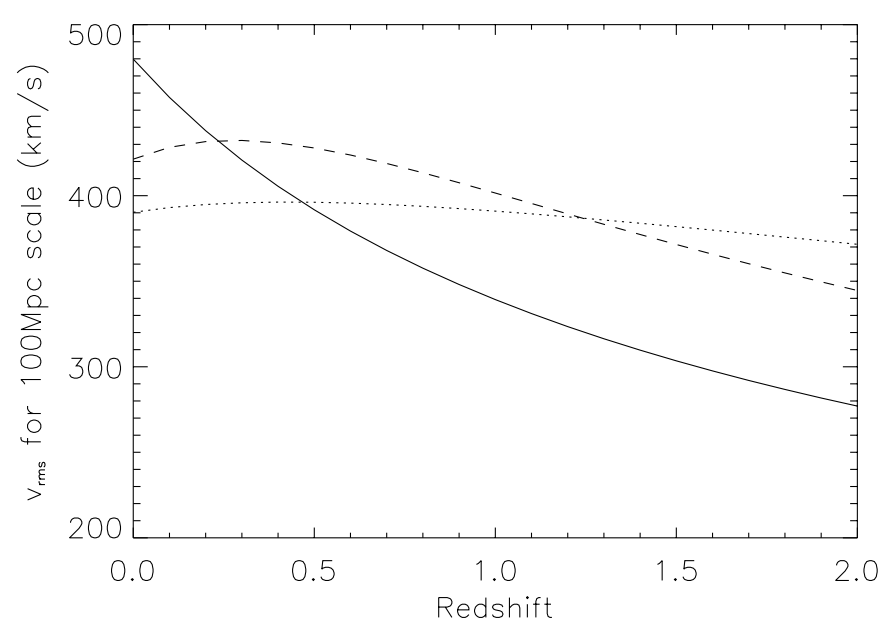

Fig. 6. The rms peculiar velocity for a $100 \mathrm{Mpc}$ scale, in linear theory, as a function of redshift for three cosmological models. The solid, dotted and dashed lines represent respectively the standard model, the open model and the flat model with a cosmological constant.

much higher, the accuracy in the bulk velocity is better than in the standard case. The overall error decreases very rapidly. It is of the order of $80 \mathrm{~km} \mathrm{~s}^{-1}$ at $z=0.5$ and reaches $30 \mathrm{~km} \mathrm{~s}^{-1}$ at $z=1$. At $z=2$, the accuracy is as small as $10 \mathrm{~km} \mathrm{~s}^{-1}$. It increases at high redshift, again when the number of clusters decreases. Similarly, in the flat model with a non-zero cosmological constant, the error in the bulk velocity decreases rapidly with redshift; but it is slightly larger than in the open case at all redshifts. The error ranges between $400 \mathrm{~km} \mathrm{~s}^{-1}$ (at low redshifts) and $25 \mathrm{~km} \mathrm{~s}^{-1}$ at $z=2$. At $z=0.5$ and $z=1$, it reaches respectively 100 and $50 \mathrm{~km} \mathrm{~s}^{-1}$. In the low density models, we thus expect to achieve very accurate measurements of the bulk motion using the SZ effect. This can be illustrated by comparing a rough estimate of $V_{\text {bulk }}$ for a $100 \mathrm{Mpc}$ scale (Fig. 6), computed in the linear theory (Eq. (11)), with the estimated error (Fig. 7). On the contrary, the precision for the standard model is rather poor $(\simeq 2 \sigma$ around $z=1)$.

These very impressive accuracies in the bulk velocity measurements would be those obtained on the $100 h^{-1} \mathrm{Mpc}$ scale using the SZ effect and assuming that all the SZ sources are detected and used for the computation. This would be the case if the sky were to be surveyed with a very high angular resolution (typically better than 1 arcmin) as will be achieved by FIRST-Herschel. In the Planck configuration, for a 5 arcmin beam convolution, source confusion will be the limiting factor in cluster detection and will lead to a degradation of the bulk velocity accuracy. Source confusion affects the measurements in all the cosmological models, but the problem is especially severe in low density matter models for which the clusters are more numerous at $z>0.3$ (e.g. Barbosa et al. 1996), as illustrated in Fig. 5. We estimate the effect of source confusion on the bulk velocity measurement, and 


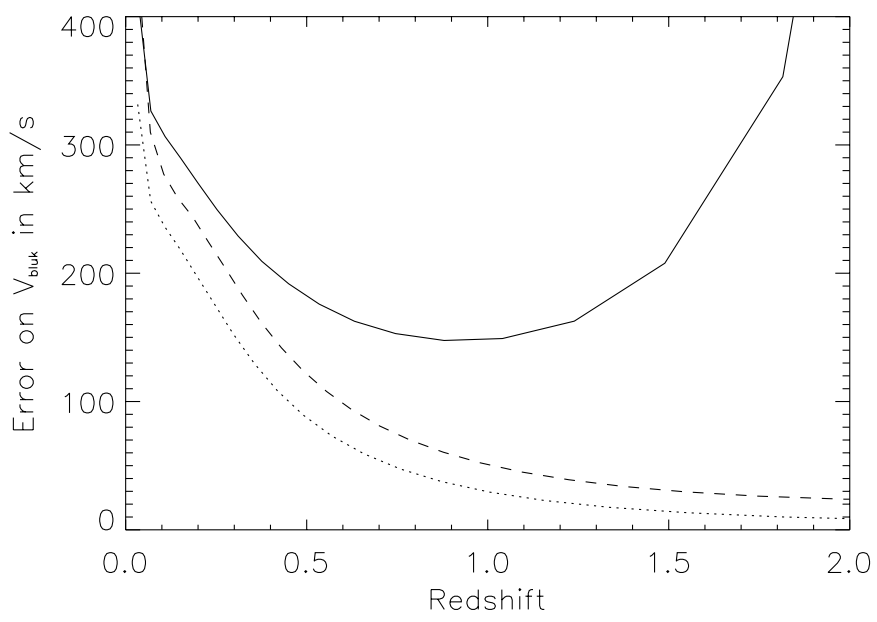

Fig. 7. The rms error in the bulk velocities measured in volumes of $100 h^{-1} \mathrm{Mpc}$ typical size. The solid line represents the standard model $\left(\Omega_{\mathrm{m}}=1\right)$, the dotted line represents the open (low $\Omega_{\mathrm{m}}$ ) model and finally the dashed line shows the results for a flat model with a non-zero cosmological constant.

illustrate the results for the low density matter models. To do so, we use the canonical confusion limit for Euclidean number counts. This implies the presence of one source in 30 independent beams ( 5 arcmin beam in the Planck configuration). Therefore, the total number of clusters on the sky must not exceed $1.9 \times 10^{5}$. This condition thus defines, for our three cosmological models, a detection (or confusion) limit $Y_{\lim }$ in terms of the integrated Compton parameter above which the clusters are detected. For the flat model with non-zero cosmological constant, we find that $Y_{\text {lim }} \sim 14 \times 10^{-3} \operatorname{arcmin}^{2}$. As expected, the limit is higher for the open model for which it is about $23 \times 10^{-3} \operatorname{arcmin}^{2}$. This comes from the fact that more clusters are predicted, and the source confusion effect is thus more important. Taking into account this additional condition in order to estimate the number of detected clusters, we re-evaluate the error in the bulk velocity in the $100 h^{-1} \mathrm{Mpc}$ typical size boxes, and we compare the results to those obtained without the correction for source confusion. The results illustrated for the two low density models are displayed in Fig. 8. The thick lines represent the errors when the limitation due to source confusion is taken into account as compared with the previous results (thin lines). In the open model (left panel), the limitation due to confusion results in a larger error, of the order of $300 \mathrm{~km} \mathrm{~s}^{-1}$ at $z=0.5$ and $50 \mathrm{~km} \mathrm{~s}^{-1}$ at $z=1$. This is, respectively, almost four times and twice as large as the previous values. In the flat model (right panel), the confusion effect increases the error in the bulk velocity by a factor of about two at $z=0.5$ where the error is about $260 \mathrm{~km} \mathrm{~s}^{-1}$. At redshift $z=1$, the error is of the order of $70 \mathrm{~km} \mathrm{~s}^{-1}$ (a factor 1.4 larger than the previous accuracy Fig. 7).
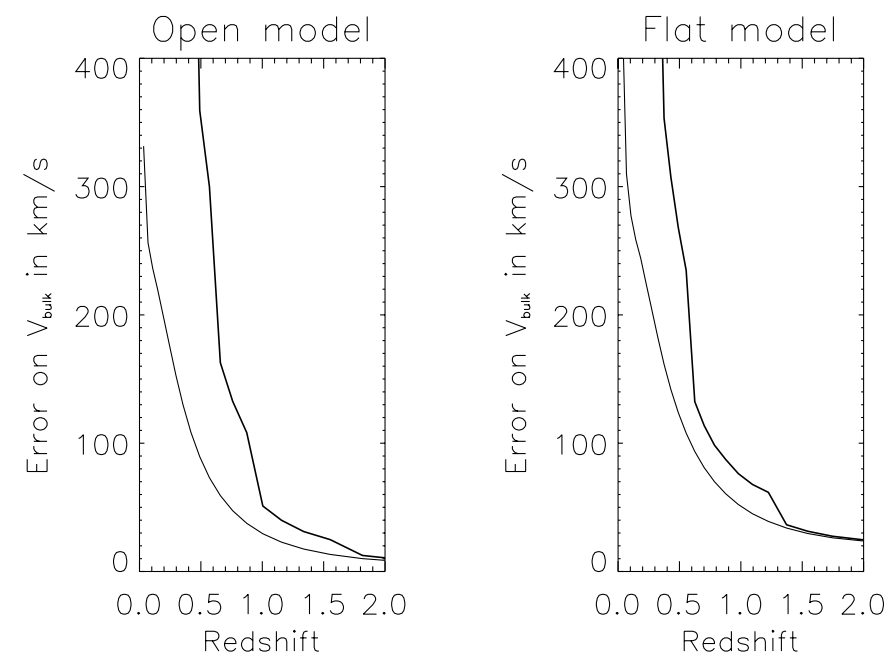

Fig. 8. The rms error in the bulk velocities $100 h^{-1}$ Mpc typical scale. The solid lines represent the errors when the limitation due to source confusion is taken into account. The thin lines shows the results uncorrected form the confusion effects (open model is in the left panel and flat model, $\Omega_{\Lambda}=0.7$, is in the right panel). The confusion limit is set so that there is one cluster in 30 independent 5 arcmin beams.

\section{Discussion and conclusion}

The kinetic SZ effect can be used as an alternative powerful tool to measure the radial component of the peculiar cluster velocities, and consequently to trace the matter distribution. Nevertheless, the accuracy for individual clusters is limited by the contributions from other astrophysical components and instrumental noise, and by the source (or spatial) confusion with other clusters. In the present study, we investigate the limitation of this method. More precisely, we estimate the rms error by which the source confusion and the major contributing sources (CMB primary anisotropies, kinetic SZ due to the background cluster population, and noise + residuals from component separation) can affect the velocity measurement.

The amplitude of the spurious cosmological contributions (CMB and SZ kinetic) and the source confusion varies with the cosmological model. Therefore, keeping the baryon density constant, we have investigated three cosmological models, two flat models with and without a cosmological constant $\left(\Omega_{\Lambda}=0\right.$ and 0.7$)$ and one open model $\Omega_{\mathrm{m}}=0.3$. A previous study by Haehnelt \& Tegmark (1996) estimated the effect of changing the baryon fraction of the universe on the error in the individual peculiar velocity.

The contribution of the kinetic SZ background clusters is not a major source of error (relative to the other contributions). It represents, depending on the cosmological model, a rms error between about 140 and $80 \mathrm{~km} \mathrm{~s}^{-1}$ for small cluster sizes, and between 50 and $30 \mathrm{~km} \mathrm{~s}^{-1}$ for more extended clusters. These errors could be up to $40 \%$ larger due to the non-linear growth of the structures, but this 
still does not significantly affect our final results because the total error is clearly dominated by the other contributions. Departures from our physical assumptions, such as possible asphericity or non-isothermality, can affect the previous values. For an individual cluster the asphericity can modify its SZ kinetic contribution by a factor 0.5 to 2 . However, this is the extreme variation for the case where the principal axis is aligned along the line of sight. In addition for a cluster population with randomly oriented ellipticities, we expect that the net geometrical effect will average out so that the error due to the background cluster population will not be significantly affected. Recent X-ray observations of galaxy clusters show isothermal profiles but still leave room for decreasing temperature profiles above $0.7 R_{\text {vir }}$. In this case, the contribution of an individual cluster is smaller especially in the cluster outskirts. As a result, the contribution to the temperature fluctuations due to the kinetic SZ effect of the whole cluster population should be smaller than in the isothermal assumption. Consequently, the departures from the simplest approximations used in our study are likely to average out, and therefore leave the final results almost unchanged. The rms error due to the CMB primary anisotropies is dominant, compared to SZ background clusters, and varies with the cosmological model and the cluster size. However, it shows the same shape in all models, i.e., large velocities at small cluster sizes (due to beam dilution effects) which sharply decrease and reach a minimum at about 2 arcmin core radius, followed by an increase at larger scales associated with the contribution from the acoustic peak. The rms error reaches its maximum value (almost $800 \mathrm{~km} \mathrm{~s}^{-1}$ ) at small cluster sizes in the open model, and its minimum value (almost $200 \mathrm{kms}^{-1}$ ) at about 2 arcmin core radius in the standard model. The last spurious component we have taken into account is that due to the residual signals from component separation including Planck like instrumental noise. This contribution is of the order of, or dominates, the CMB depending on the amplitude of the cosmological "errors" (that is depending on the cosmological model). The induced rms error shows a maximum value for large cluster sizes, where it reaches about $1000 \mathrm{~km} \mathrm{~s}^{-1}$, it decreases to $400 \mathrm{~km} \mathrm{~s}^{-1}$ at 2 arcmin core radius then increases again up to $500 \mathrm{~km} \mathrm{~s}^{-1}$. The contribution from residuals in the component separation could well be smaller if we use betteradapted component separation techniques, such as the proposed maximum entropy method (Hobson et al. 1998). All the resulting accuracies are obtained for a rather important thermal effect $\left(y_{0}=10^{-4}\right)$. The velocities vary as a function of $1 / y_{0}$, and "weaker" clusters are affected by larger errors. Another source of error in the peculiar velocity is the relativistic correction to the SZ effect, which is not taken into account in our study. It has been evaluated by Holzapfel et al. (1997) who found it to be of the order of $360\left(k T_{\mathrm{e}} / 10 \mathrm{keV}\right)^{2} \mathrm{~km} \mathrm{~s}^{-1}$. Furthermore, our results do not take into account the error due to the uncertainty in the measured intra-cluster temperature. As previously mentioned, the recent X-ray satellite observations will allow very precise temperature measurements with an accuracy of 5 to $10 \%$.

The measurement of the radial component of the peculiar cluster velocity using the SZ effect is only marginally possible for the "strongest" clusters, and then only in a rather narrow spatial window corresponding to clusters with core radii around 2 arcmin. The number of clusters matching the two criteria (size and amplitude) over the sky is small. Therefore, we rather turn to a statistical approach. The velocity dispersion is not the appropriate quantity to study as it is very sensitive to systematic effect contributions. The bulk velocity, in turn, is a betteradapted statistical quantity which can be derived from the SZ measurement of the individual peculiar velocities. Based on the all-sky high sensitivity SZ survey which will be provided by the Planck mission, we propose a strategy to trace the velocity field by measuring the bulk flows on very large scales up to $z=1$, or greater. The method relies on averaging, over large volumes, the peculiar velocities of the individual clusters detected therein. In this context, we have evaluated the rms error associated with the bulk velocity in $100 h^{-1} \mathrm{Mpc}$ typical size boxes by generalising, to a population of clusters predicted by the PS formalism, the results obtained for individual clusters. The accuracy in the bulk velocity $\left(\sigma_{\text {bulk }}\right)$ depends on two main quantities: the accuracy in the individual radial velocities $\left(\sigma_{i}\right)$ and the number of clusters $\left(N_{i}\right)$. The smaller $\sigma_{i}$ then the smaller is $\sigma_{\text {bulk }}$. The larger $N_{i}$ then the smaller is $\sigma_{\text {bulk }}$. Therefore, we find a rather poor accuracy in the standard flat model for which the predicted number of clusters is small. In contrast, in both low matter density models $\left(\Omega_{\mathrm{m}}<1\right)$, the accuracy in the bulk velocities is higher. In our study, we find that the accuracy of the bulk flow determination is thus dominated by the numerous low mass clusters. However, large cluster numbers result in source confusion which in turn limits our ability to detect individual clusters. It thus decreases the number of clusters useful for the bulk velocity estimates which results in a larger $\sigma_{\text {bulk }}$. Taking into account the fact that source confusion will prevent us from detecting all the individual SZ clusters in the Planck all-sky survey, we have estimated the new errors on the bulk velocities (Fig. 8). Our method cannot be applied for $100 h^{-1}$ Mpc boxes, due to lack of sources above the detection limit, below a redshift of 0.15 and 0.35 in, respectively, the flat and open models. In the flat model, the errors are increased with decreasing redshifts by a factor reaching 3 at $z=0.22$. In the open model, the degradation due to source confusion is even stronger. The degradation factor is 2.75 at $z=0.66$ increasing to 4 at $z=0.5$. There is almost no loss of accuracy at $z \geq 1.8$. In the low density models, the bulk velocities measured with the SZ effect exhibit large errors at small redshifts and small errors at high redshifts. These results show that the the bulk velocities on large scales, especially at high redshifts, can be accurately measured and mapped through the SZ effect (see Figs. 6 and 8). This is opposite to the case of classical velocity measurements where the errors are larger at higher redshifts 
(e.g. Willick 1999 and references therein). The velocity field could thus be mapped using these two complementary approaches (traditional and SZ) as a function of the redshift range that is probed.

Assuming a low matter density universe $\left(\Omega_{\mathrm{m}}=0.3\right)$, a proposed strategy to measure the bulk velocities on large scales using the SZ effect would be to average the individual peculiar velocities in large boxes between redshifts of 0 and 1 , or more. The size of the boxes would be chosen so that the obtained accuracy in the bulk velocity is of the order of a few tens $\mathrm{km} \mathrm{s}^{-1}$. This allows an inhomogeneous sampling of the universe. A sampling with $100 h^{-1} \mathrm{Mpc}$ typical size boxes gives satisfying accuracy around $z=1$ $\left(\sigma_{\text {bulk }} \sim 80-50 \mathrm{~km} \mathrm{~s}^{-1}\right)$, but gives larger errors around $z=0.5$ especially in an open model. One way of overcoming this problem would be to increase the statistical cluster sample by estimating the bulk velocity on larger scales $\left(>100 h^{-1} \mathrm{Mpc}\right)$ at intermediate redshifts. Another possibility would be to measure the bulk velocity of the local universe up to a certain redshift, typically $z=0.2$ for flat universe and $z=0.4$ for open universe. In order to measure the velocities using the $\mathrm{SZ}$ effect, we need, in addition to a measurement of the Compton parameter $y$ and the temperature fluctuation of each cluster $\delta T / T$, its electron intra-cluster temperature $T_{\mathrm{e}}$ and its redshift $z$. At intermediate redshift $(0<z<0.5)$, we could use for a very large fraction of the sky (about $\pi$ steradian), the SDSS redshift determinations and the available X-ray observations (ROSAT, ASCA, XMM-Newton, Chandra) to derive the temperature. The temperature could be obtained either through spectroscopic measurements, or through empirical relations with general properties established on smaller samples. At higher redshifts $(0.5<z<1)$, we could focus on a few selected regions of the sky. The regions in this case, should correspond to sky selected areas on which multi-wavelength observations will provide cluster surveys with their redshifts and temperatures or masses (VIRMOS, XMM-Newton, Chandra, MEGACAM). For a better sky coverage, specific complementary observations could be programmed before the Planck mission.

The study of galaxy cluster peculiar velocities is advantageous since, on scales probed by clusters, the underlying density fluctuations are largely in the linear regime and therefore very close to the initial conditions from which large-scale structure developed. The large-scale velocity field represents a direct prediction of the cosmological model and depends on the power spectrum and matter density. Following our proposed strategy, based on the all-sky SZ survey provided by Planck, we will be able to study the velocity field over cosmologically important scales and examine its evolution. We could use, as proposed by Ferreira et al. (1999) and Juszkiewicz et al. (1999), the evolution of the mean relative velocity of pairs of boxes as a function of their separation to directly constrain the cosmological parameters. We could also apply methods based on the comparison between the reconstructed total matter distribution from velocity fields (Bertschinger \& Dekel 1989; Zaroubi et al. 1999) and that traced by galaxies to constrain the matter density; the constraints on the power spectrum over these large scales can be directly compared to CMB constraints.

Acknowledgements. The authors would like to thank M. Arnaud, C. Balland and J. Bartlett for interesting discussions, and A. Jones for his very careful reading. They also thank an anonymous referee for helpful comments.

\section{References}

Aghanim, N., De Luca, A., Bouchet, F. R., Gispert, R., \& Puget, J. L. 1997, A\&A, 325, 9

Arnaud, M., et al., in Clusters of galaxies and the high redshift universe observed in X-rays, Rencontres de Moriond, 2001, ed. D. Neumann, \& J. T. T. Van

Bahcall, N. A., Cen, R., \& Gramann, M. 1994, ApJ, 430, L13

Barbosa, D., Bartlett, J. G., Blanchard, A., \& Oukbir, J. 1996, A\&A, 314, 13

Bertschinger, E., \& Dekel, A. 1989, ApJ, 336, L5

Birkinshaw, M., \& Gull, S. F. 1983, Nature, 302, 315

Birkinshaw, M., Hughes, J. P., \& Arnaud, K. A. 1991, ApJ, 379,466

Birkinshaw, M. 1983, in Quasars \& Gravitational Lenses: 24th Liège, 134

Bouchet, F. R., \& Gispert, R. 1999, New Astron., 4, 443

Bryan, G. L., \& Norman, M. L. 1998, ApJ, 495, 80

Carroll, S. M., Press, W. H., \& Turner, E. L. 1992, ARA\&A, 30,499

Cavaliere, A., \& Fusco-Femiano, R. 1978, A\&A, 70, 677

Challinor, A., \& Lasenby, A. 1998, ApJ, 499, 1

Colberg, J. M., White, S. D. M., Macfarland, T. J., et al. 2000, MNRAS, 313, 229

Courteau, S., Faber, S. M., Dressler, A., \& Willick, J. A. 1993, ApJ, 412, L51

Dressler, A., Faber, S. M., Burstein, D., et al. 1987, ApJ, 313, L37

Edge, A. C., \& Stewart, G. C. 1991, MNRAS, 252, 414

Evrard, A. E. 1990, ApJ, 363, 349

Faber, S. M., \& Jackson, R. E. 1976, ApJ, 204, 668

Ferreira, P. G., Juszkiewicz, R., Feldman, H. A., Davis, M., \& Jaffe, A. H. 1999, ApJ, 515, L1

Giovanelli, R., Haynes, M. P., Wegner, G., et al. 1996, ApJ, 464, L99

Giovanelli, R., Haynes, M. P., Salzer, J. J., et al. 1998, AJ, 116, 2632

Haehnelt, M. G., \& Tegmark, M. 1996, MNRAS, 279, 545

Hansen, F. K., \& Lilje, P. B. 1999, MNRAS, 306, 153

Hobson, M. P., Jones, A. W., Lasenby, A. N., \& Bouchet, F. R. 1998, MNRAS, 300, 1

Holzapfel, W. L., Ade, P. A. R., Church, S. E., et al. 1997, ApJ, 481, 35

Hudson, M. J., Smith, R. J., Lucey, J. R., et al. 1999, ApJ, $512, \mathrm{~L} 79$

Hudson, M. J. 1994, MNRAS, 266, 475

Hughes, J. P., \& Birkinshaw, M. 1998, ApJ, 501, 1

Itoh, N., Kohyama, Y., \& Nozawa, S. 1998, ApJ, 502, 7

Jones, C., \& Forman, W. 1984, ApJ, 276, 38

Juszkiewicz, R., Springel, V., \& Durrer, R. 1999, ApJ, 518, L25

Kashlinsky, A., \& Atrio-Barandela, F. 2000, ApJ, 536, L67

King, I. R. 1966, AJ, 71, 64

Lahav, O., Rees, M. J., Lilje, P. B., \& Primack, J. R. 1991, MNRAS, 251, L128 
Lamarre, J. M., Giard, M., Pointecouteau, E., et al. 1998, ApJ, 507, L5

Markevitch, M., Vikhlinin, A., Mazzotta, P., \& Vanspeybroeck, L. 2000, in astronomy 2000 (Palermo), X, ed., R. Giacconi, L. Stella, \& S. Serio. ASP Conf. Ser.

Molnar, S. M., \& Birkinshaw, M. 1999, ApJ, 523, 78

Moscardini, L., Branchini, E., Brunozzi, P. T., et al. 1996, MNRAS, 282, 384

Naselsky, P., Novikov, D., \& Silk, J. 2000 [astro-ph/0007133]

Nozawa, S., Itoh, N., Kawana, Y., \& Kohyama, Y. 2000, ApJ, 536, 31

Peebles, P. J. E. 1980, in The large-scale structure of the Universe

Peebles, P. J. E. 1993, in Principles of physical cosmology

Pointecouteau, E., Giard, M., \& Barret, D. 1998, A\&A, 336, 44

Press, W. H., \& Schechter, P. 1974, ApJ, 187, 425
Rephaeli, Y., \& Lahav, O. 1991, ApJ, 372, 21

Rephaeli, Y. 1995, ARA\&A, 33, 541

Sazonov, S. Y., \& Sunyaev, R. A. 1998, ApJ, 508, 1

Scherrer, R. J., Melott, A. L., \& Shandarin, S. F. 1991, ApJ, 377,29

Sunyaev, R. A., \& Zel'dovich, I. B. 1980, ARA\&A, 18, 537

Tully, R. B., \& Fisher, J. R. 1977, A\&A, 54, 661

Viana, P. T. P., \& Liddle, A. R. 1996, MNRAS, 281, 323

Viana, P. T. P., \& Liddle, A. R. 1999, MNRAS, 303, 535

Walker, T. P., Steigman, G., Kang, H. S., Schramm, D. M., \& Olive, K. A. 1991, ApJ, 376, 51

Willick, J. A., Courteau, S., Faber, S. M., Burstein, D., Dekel, A., \& Kolatt, T. 1996, ApJ, 457, 460

Willick, J. A. 1999, ApJ, 522, 647

Wright, E. L. 1979, ApJ, 232, 348

Zaroubi, S., Hoffman, Y., \& Dekel, A. 1999, ApJ, 520, 413 